\title{
NUCLEAR MATTER INCOMPRESSIBILITY FROM A SEMI-EMPIRICAL ANALYSIS OF BREATHING-MODE ENERGIES
}

\author{
M.M. SHARMA' and W. STOCKER \\ Sektion Physik, Universität München, D-8046 Garching, Fed. Rep. Germany \\ P. GLEISSL and M. BRACK
}

Institut für Theoretische Physik, Universität Regensburg, D-8400, Regensburg, Fed. Rep. Germany

Received 4 January 1989

(Revised 12 June 1989)

\begin{abstract}
We check the validity and applicability of a liquid-drop model type expansion for the incompressibility $K_{\mathrm{A}}$ of finite nuclei: $K_{\mathrm{A}}=K_{\mathrm{V}}+K_{\mathrm{S}} A^{1 / 3}+$ (higher-order terms). Our theoretical considerations are based upon calculations of breathing-mode energies following from a density variational framework taking into account various Skyrme interactions. Using a semi-empirical procedure based upon this expansion of $K_{\mathrm{A}}$, we corroborate that new precision data for the monopole energies favour a volume coefficient $K_{\mathrm{V}} \sim(300 \pm 25) \mathrm{MeV}$ and an appreciable surface coefficient $K_{\mathrm{S}} \sim(-750 \pm 80) \mathrm{MeV}$. We discuss the implication of this result for the incompressibility $K_{\mathrm{x}}$ of infinite nuclear matter.
\end{abstract}

\section{Introduction}

The nuclear matter incompressibility $K_{x}$ is an important ingredient in the nuclear equation of state. Its value enters into analyses of astrophysical phenomena as well as of low- and high-energy heavy-ion reactions. Whereas hydrodynamical simulations of prompt explosions of supernovae ') require a rather low value of $K_{x} \leqslant$ $180 \mathrm{MeV}$, neutron-star masses ${ }^{2}$ ) favour a much larger value of about $340 \mathrm{MeV}$. Skyrme interactions reproducing nuclear ground-state properties and fission barriers ${ }^{3}$ ) have $K_{x}$ values around $220 \mathrm{MeV}$. In order to yield charge-density differences of isotopes, $\mathrm{Co}^{*}$ and Speth ${ }^{4}$ ) used a Migdal-type potential with $K_{x^{-}} \sim 350 \mathrm{MeV}$. The same data were reproduced by Cavedon et al. ${ }^{5}$ ) using the density-dependent Gogny interaction with an incompressibility $K_{x}$ of about $220 \mathrm{MeV}$. Bartel et al. ${ }^{6}$ ) concluded that the charge-density difference between neighbouring $\mathrm{Pb}$ isotopes is appreciably influenced by nuclear parameters other than $K_{x}$. High-energy heavy-ion data ${ }^{7}$ ) favour a rather high value of $K_{x}$ around $400 \mathrm{MeV}$. Since $K_{x}$ is defined from the second derivative of the energy per particle in nuclear matter with respect to the density at the point of saturation, the most direct way to the empirical value of $K_{x}$ goes via an analysis of small amplitude oscillations around saturation density, i.e.

' Fellow of the Alexander von Humboldt Foundation, Fed. Rep. Germany. 
of the breathing mode in finite nuclei. But only nuclei with $A<250$ can be experimentally investigated, and thus the extraction of the value $K_{x}$ for the (hypothetical) infinite homogeneous symmetric nuclear matter is still indirect and is, in fact, a highly non-trivial problem.

Based upon microscopic HF + RPA calculations for various zero- and finite-range effective nuclear interactions, Blaizot et al. ${ }^{8}$ ) claimed $K_{x}=210+30 \mathrm{MeV}$ in order to reproduce the pioneering experimental results of Marty et al. ${ }^{9}$ ) for the breathingmode energies of the nuclei ${ }^{410} \mathrm{Ca},{ }^{90} \mathrm{Zr}$ and ${ }^{208} \mathrm{~Pb}$. Similarly, Bohigas et al. ${ }^{10}$ ) analyzed both nuclear monopole and quadrupole giant resonances in terms of RPA sum rules, evaluated for a wide range of Skyrme forces, and found $K_{x} \simeq 230 \mathrm{MeV}$. In a detailed microscopic HF+RPA analysis using various Skyrme interactions, Blaizot ${ }^{11}$ ) finally determined $K_{a}=210 \pm 30 \mathrm{MeV}$, based upon the comparison with the experimental data known at the time, in particular of ${ }^{208} \mathrm{~Pb}$ and ${ }^{90} \mathrm{Zr}$.

Since the datum used ${ }^{11}$ ) for ${ }^{90} \mathrm{Zr}$ is questionable ${ }^{12}$ ) and since many new precise empirical data of breathing-mode energies are now available ${ }^{13}$ ), we propose in this paper to re-analyze the semi-phenomenological method, first outlined by Pandharipande ${ }^{14}$ ) and later applied by Treiner et al. ${ }^{15}$ ), based upon a liquid-drop model (LDM) type expansion of the finite-nucleus incompressibility $K_{\mathrm{A}}$.

\section{The nuclear incompressibility $\boldsymbol{K}_{\mathrm{A}}$}

There is no unique definition of the incompressibility $K_{\mathrm{A}}$ of a finite nucleus. It might be obtained ${ }^{16}$ ) as the second derivative of the energy per particle with respect to the radius parameter $R$ at the equilibrium value $R_{0}$

$$
K_{\mathrm{A}}^{R}=\left.R_{0}^{2} \cdot \frac{\partial^{2}(E / A)}{\partial R^{2}}\right|_{R_{0},},
$$

in order to evaluate this expression one has, however, to know the dependence of the energy $E$ on the radius $R$. Alternatively, a scaling transformation $r \rightarrow \lambda r$, applied to the single-particle wavefunctions of the HF ground state, leads to the so-called "scaling incompressibility"

$$
K_{A}^{\text {scal }}=\left.\frac{\partial^{2}(E / A)}{\partial \lambda^{2}}\right|_{\lambda-1} .
$$

Estimating the breathing-mode energy by $E_{k}=\sqrt{m_{k} / m_{k-2}}$ in terms of the RPA sum rules $m_{k}$ evaluated with the isoscalar excitation operator $\hat{Q}=r^{2}$ - whose form is only a guess - leads to expressions of the form ${ }^{10}$ )

$$
E=\sqrt{\frac{\hbar^{2} K_{\mathrm{A}}}{m\left\langle r^{2}\right\rangle}},
$$

where $\left\langle r^{2}\right\rangle$ is the mean square radius of the HF ground state and $m$ is the nucleon mass. For $k=3$ one obtains $K_{\mathrm{A}}=K_{\mathrm{A}}^{\text {scal }}$, eq. (2), and for $k=1$ one is led to a "constrained incompressibility" for $K_{\mathrm{A}}$ which can be identified with $K_{\mathrm{A}}^{R}$, eq. (1), 
if $R_{0}=\left\langle r^{2}\right\rangle^{1 / 2}$ and the $R$-dependence of the HF energy is obtained by a constrained HF calculation with $\hat{Q}=r^{2}$ as an external field.

In hydrodynamical terminology, the breathing-mode energy is given by $E=$ $\hbar \sqrt{C / B}$ in terms of a restoring-force parameter $C$ and an inertial parameter $B$. These quantities depend on the nature of the density vibration which might be characterized by the velocity (or displacement) field or the associated transition density. They are, in particular, sensitive to the way in which the nuclear surface is coupled to the nuclear bulk during the breathing-mode oscillation. In our analysis, based upon a density variational approach ${ }^{17-19}$ ) to be briefly summarized in sect. 4 , we assume that a nucleus, even in a state of compression or decompression during the monopole vibration, still displays a division into a homogeneous bulk part and a surface skin ("leptodermous" behaviour). Scaling is one special type in this whole class of density oscillations, and only in this particular case can the inertial parameter $B$ be identified with $m\left\langle r^{2}\right\rangle$, leading to eq. (3) with $K_{\mathrm{A}}=K_{\mathrm{A}}^{\text {scal }}$. Several microscopic calculations ${ }^{8,11.15}$ ) have, indeed, shown that RPA transition densities in heavy nuclei are similar to those obtained in the scaling model. There exists, however, no general theoretical argument for believing that the scaling assumption and thus eq. (3) for the breathing-mode energy is correct. Only detailed empirical information about the transition density, which is still lacking, would allow to determine the exact form of the inertial parameter.

\section{Leptodermous expansion of $K_{A}$}

The strategy of our semi-empirical analysis is the following. We assume that the experimental breathing-mode energies (strictly speaking, the centroids of the resonance peaks) are given by expression (3), and we then use the empirical values of $\left\langle r^{2}\right\rangle$ for extracting $K_{A}$. Insofar, our analysis seems to be restricted to the scaling type vibration. However, for $K_{\mathrm{A}}$ we now assume a LDM expansion ${ }^{14.15 .20}$ ) to exist

$$
K_{\mathrm{A}}=K_{\mathrm{V}}+K_{\mathrm{S}} A^{1 / 3}+K_{2}\left(\frac{N-Z}{N+Z}\right)^{2}+K_{\text {coul }} Z^{2} A^{-4 / 3}+K_{\text {curv }} A^{-2 / 3},
$$

and determine the parameters in (4) by a least-squares fit of the experimental breathing-mode energies to eq. (3). Expansion (4) need not be based a priori on the scaling assumption, nor on other approximations such as mean-field theory with Skyrme type interactions. It may therefore supply a semi-phenomenological basis for a fit of its constants to empirical data in a way analogous to the procedure for the semi-empirical mass formula. That the eqs. (3) and (4) allow, indeed, to extract correctly the parameter $K_{\mathrm{v}}$ even in a theoretical model which goes beyond the scaling assumption and in which the collective inertia is not proportional to $m\left\langle r^{2}\right\rangle$, will be demonstrated below (table 1).

The connection of the volume parameter, $K_{\mathrm{V}}$, to the nuclear matter incompressibility, $K_{x}$, depends on the particular nature of the breathing-mode oscillation. In the 
scaling case, one finds $\left.{ }^{10,16,20}\right) K_{\mathrm{V}}=K_{\infty}$. However, in the "adiabatic" density compression mode where the mean-square radius is constrained, leading to $K_{A}^{R}$, eq. (1), the "leptodermous" separation of the breathing density into a homogeneous bulk and a thin surface region is no more possible, and as a consequence, it was found ${ }^{15.16}$ ) that expansion (4) converges poorly and the volume term becomes, in fact, $K_{\mathrm{V}}=\frac{7}{10} K_{x}$.

In the scaling case, all the coefficients of expansion (4) can be uniquely determined and related to the results of infinite or semi-infinite nuclear matter calculations ${ }^{15,20}$ ). It was shown ${ }^{11}$ ) how the theoretical surface coefficient $K_{\mathrm{S}}$ depends on the second derivative $\ddot{\sigma}$ of the nuclear surface tension with respect to the central density. The dependence of $\ddot{\sigma}$ on the coupling of surface and bulk vibrations was studied in ref. ${ }^{17}$ ) using a simple model energy density. For a static compression the lowestenergy state was obtained for an anti-scaled density. A hydrodynamical calculation ${ }^{18}$ ) later revealed that dynamical effects are responsible for the shifting of the mode to the scaling-type behaviour, as found also in the microscopic RPA calculations. In a refined version of this hydrodynamical model ${ }^{19}$ ), which also takes into account the asymmetry of the surface density profile $\left(\gamma_{q} \neq 1\right.$ in eq. (7) below), the parameter $\beta$ describing the coupling of surface to the bulk (see eq. (8) below) turned out to depend sensitively on the mass number $A$. Such a behaviour destroys to some extent the meaning of the expansion coefficients in eq. (4), since it implies that the surface contribution to $K_{\mathrm{A}}$ depends on $A$ and would have to be further expanded in order to yield unique coefficients. For vibrational modes with $\beta$ practically independent of $A$ (but not necessarily of scaling type), expansion (4) should converge sufficiently well and then serve as a basis for the determination of $K_{\mathrm{V}}$.

An analytical expression ${ }^{11}$ ) for $K_{\text {(oul }}$ in eq. (4) is

$$
K_{\text {coul }}=\frac{3}{5}\left(e^{2} / r_{\mathrm{c}}\right)(1-27 R) \text {. }
$$

The quantity $R$ is related to the third derivative of the bulk energy density $\varepsilon$ with respect to the bulk density as given by

$$
R=\frac{\rho_{0}^{2}}{K_{x}} \frac{\mathrm{d}^{3} \varepsilon}{\mathrm{d} \rho_{0}^{3}} .
$$

This expression for $K_{\text {coul }}$ differs from that originally proposed by Pandharipande ${ }^{14}$ ). The determination of an empirical value of the third derivative in eq. (6) goes beyond that of $K_{\mathrm{V}}$ itself. It was shown ${ }^{15}$ ) that for a class of Skyrme interactions the quantity $R$ in eq. (6) can be approximated by $R=0.5-45(\mathrm{MeV}) / K_{\mathrm{v}}$. In our analysis we take $K_{\text {coul }}$ to be given by eq. (5) using this approximation of $R$. Since $K_{\mathrm{S}}$ depends on the second derivative of the surface tension and therefore on the empirically unknown coupling between surface and bulk, it must be determined through the fit to experimental resonance energies. The asymmetry coefficient $K_{\Sigma}$ is also determined by the fit. The curvature term in eq. (4) is the least significant one; its determination with other parameters depends on the number of the data 
available for the fit. In fitting a limited number of experimental data, we have, therefore, fixed $K_{\text {curv }}$ (see table 3 ).

It might at first sight seem problematic to try to determine the parameters in eq. (4) by a fit to a limited number of nuclei with $A<250$, since expansion (4) is asymptotic in its nature, i.e. valid only in the limit of very large values of $A$. This misgiving is even more justified considering the fact that the absolute values of the coefficients in (4) converge much less than e.g. those of the nuclear mass formula. It is therefore one of the purposes of this paper to demonstrate, with the help of realistic but theoretically calculated values of $K_{\mathrm{A}}$, that the fitting procedure using a relatively limited number of sample nuclei indeed makes sense and is able to correctly extract the coefficients $K_{\mathrm{V}}$ and $K_{\mathrm{S}}$, which are known a priori in the scaling model used.

\section{Model calculations}

We start from a density variational calculation of the average nuclear binding energy, using the semiclassical local density functionals of the extended ThomasFermi (ETF) model in connection with Skyrme-type effective interactions. [See ref. $^{3}$ ) for the details of these ETF variational calculations.] The spherical neutron and proton density profiles are hereby parametrized by

$$
\rho_{q q}(r)=\rho_{0 q q}\left[1+\exp \left(\frac{r-R_{q q}}{\alpha_{q}}\right)\right]^{-\gamma_{q u}}, \quad(q=\mathrm{n}, \mathrm{p})
$$

and the total energy is minimized with repect to the 8 parameters in (7), keeping the nucleon numbers $Z$ and $N$ fixed. The breathing-mode energies are then calculated in an extended hydrodynamical model $\left.{ }^{18,19}\right)$, taking the density parameters $\rho_{90,4}$ and $\alpha_{q}$ as time-dependent collective variables (the radii $R_{q}$ are adjusted at any time to conserve the nucleon numbers $Z, N$ and $\gamma_{q}$ is kept constant). The isoscalar nature of the breathing mode is imposed by letting the proton and neutron parameters oscillate in phase. By solving the continuity equations numerically, the velocity fields corresponding to these bulk density and surface vibrations are determined, and from them the inertial tensor is found. The restoring force tensor is directly determined by numerical differentiation of the total energy with respect to the collective parameters. Finally, the system of coupled harmonic oscillators (bulk and surface oscillations) is diagonalized. [See refs. ${ }^{18,19}$ ) for the details.] The lower of the two eigenmodes is found to be very close to the experimental breathing-mode energy, in particular for nuclei with $A>150$ (see also fig. 1 below); for heavy nuclei it contains $=100 \%$ and for the lightest nuclei less than $50 \%$ of the collective $r^{2}$ strength, in good agreement with experiments (see the detailed discussion of the results in ref. $\left.{ }^{19}\right)$ ). It should be mentioned that these results were obtained with a Skyrme force $\left.{ }^{3}\right)\left(\right.$ SkM $\left.^{*}\right)$ with $K_{x}=217 \mathrm{MeV}$. 
The dynamical coupling of the parameters $\rho_{0 q}$ and $\alpha_{q}$ may be described ${ }^{17}$ ) in terms of a coupling parameter $\beta$ defined by

$$
\frac{\alpha_{q}(t)}{\alpha_{q}(0)}=\left[\frac{\rho_{0 q}(t)}{\rho_{0 q}(0)}\right]^{\beta} \text {. }
$$

The scaling mode described above, which leads to a restoring force parameter equal to $K_{\mathrm{A}}^{\text {scal }}$, eq. (2), and an inertial parameter equal to $m\left\langle r^{2}\right\rangle$, is obtained if $\beta$ is fixed to the value $\beta=-\frac{1}{3}$. The LDM coefficients of $K_{A}^{\text {scal }}$ converge weakly but still fast enough, so that the numerically obtained values of $K_{\mathrm{A}}^{\text {scal }}$ can be recalculated via eq. (4) with sufficient accuracy even for light nuclei. (E.g. the next missing term in (4) of order $A^{-1}$ is practically zero. See also an extensive discussion of the convergence of the LDM expansion for $K_{\mathrm{A}}^{\text {scal }}$ in ref. ${ }^{20}$ ). .)

In the two-dimensional hydrodynamical model ${ }^{19}$ ), all coefficients in (4) but $K_{\mathrm{v}}$ depend on the coupling parameter $\beta$ which, as discussed above, varies strongly with $A$ from medium to light nuclei. Due to the underlying "leptodermous" assumption of the breathing nuclear densities in the model ${ }^{19}$ ), the volume coefficient $K_{\mathrm{V}}$ is, however, still identical ${ }^{17,18}$ ) with $K_{\infty}$.

\section{Theoretical and experimental data}

First, we show in fig. 1 the calculated and measured breathing-mode energies of the nuclei included in our fits. The crosses in the upper part of fig. 1 show the results

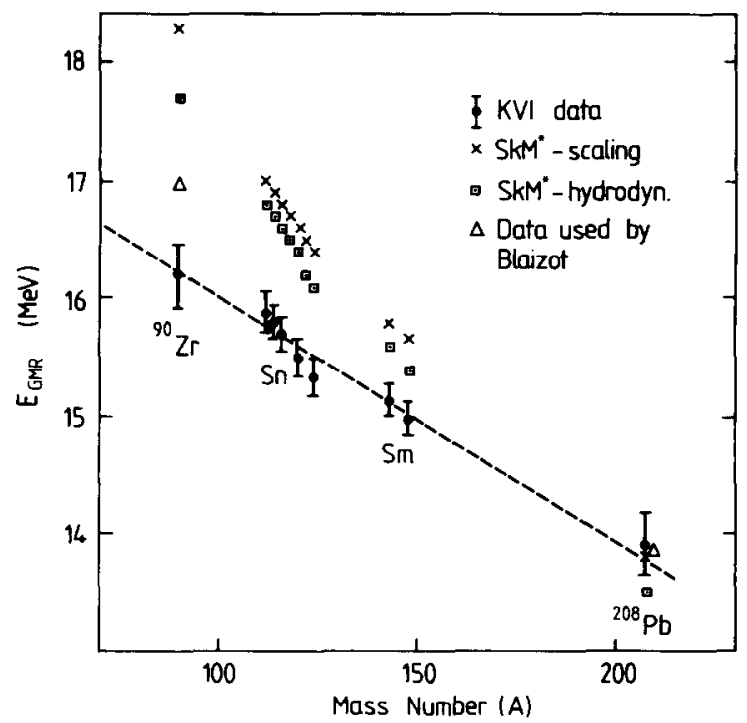

Fig. 1. Nuclear breathing-mode energies. Experimental giant-monopole-resonance (GMR) data from KVI, Groningen ${ }^{12,13,21}$ ). Theoretical $\mathrm{SkM}^{*}$ results are from ref. ${ }^{19}$ ) (see also the text). The dashed line has been drawn only to guide the eye. 
of scaling ${ }^{19}$ ) (i.e., the RPA energies $E_{3}$ for the $r^{2}$ operator, evaluated from the HF ground-state wave functions), whereas the dotted squares represent the energies obtained in the two-dimensional hydrodynamical approach ${ }^{19}$ ) (both obtained with the Skyrme force $S k M^{*}$ ). The experimental data (with error bars) have been taken from recent KVI-Groningen work ${ }^{12.13 .21}$ ). The dashed line has been drawn only to guide the eye along the experimental results. The data shown by triangles for ${ }^{2018} \mathrm{~Pb}$ and ${ }^{90} \mathrm{Zr}$ were used by Blaizot ${ }^{11}$ ) in his analysis. Clearly, the calculations with $\mathrm{SkM}^{*}$ overestimate the data for the medium-mass nuclei by about $0.4-2 \mathrm{MeV}$. For ${ }^{200} \mathrm{~Pb}$, they come very close to the experimental value ${ }^{21}$ ). In ref. ${ }^{19}$ ), the results of the two-dimensional hydrodynamical model have been shown to be in good agreement with the existing experimental data for all nuclei; the measured GMR energies are overestimated on the average by only $\simeq 1-2 \mathrm{MeV}$. The microscopically evaluated HF-RPA scaling energies $E_{3}$, however, were found ${ }^{19}$ ) to overestimate the hydrodynamical and thus also the experimental energies for light nuclei $(A<30)$ by up to $60 \%$. This indicates that the collective $r^{2}$ RPA strength is strongly fragmented in those light nuclei and that the measured GMR energies correspond, indeed, to the lower of the hydrodynamically obtained states which carries $50 \%$ (or less) of the strength $\left.{ }^{19}\right)$.

\subsection{FITS TO THEORETICAL DATA}

We now discuss the results of our fitting procedure to determine $K_{\mathrm{V}}$ and $K_{\mathrm{S}}$, employing the theoretical results for $K_{\mathrm{A}}$ discussed above. In tables 1 and 2 we display the values of the coefficients $K_{\mathrm{V}}, K_{\mathrm{S}}, K_{\Sigma}$ and $K_{\text {curv }}$ for the scaling case, obtained (i) by the exact asymptotic expansion and (ii) from various fits to eq. (4) for the Skyrme interactions $\mathrm{SkM}^{*}$ (table 1) and SIII (table 2), as well as those obtained by various fits to the hydrodynamical ${ }^{19}$ ) values of $K_{\mathrm{A}}$ for $\mathrm{SkM}^{*}$ (table 1 ). The last columns contain the values of the relative $\chi^{2}$ per degree of freedom (for its description see e.g. ref. ${ }^{24}$ )). In order to compare the quality of fits to the theoretical data with those of experimental data, we choose the error bars in the theoretical breathing-mode energies to be comparable to the experimental ones ${ }^{13}$ ). This corresponds to about $\pm 150 \mathrm{keV}$ for all nuclei from ${ }^{112} \mathrm{Sn}$ to ${ }^{208} \mathrm{~Pb}$ and to $\pm 200 \mathrm{keV}$ for ${ }^{90} \mathrm{Zr}$. Actually, this represents an upper bound on the theoretical error bars; the purely numerical uncertainty in the theoretical values of $K_{A}$ is much smaller $(\sim 0.01 \%)$. Since $\chi^{2}$ goes as the inverse squared error bar, this reduces $\chi^{2}$ for a best fit to considerably less than unity, and therefore the $\chi^{2}$ shown in the tables are relative $\chi^{2}$ which depend on the assumed error bars. The same is also true for the $\chi^{2}$ values shown in table 3 for the fits on experimental data. The error bars in the values of $K_{\text {A }}$ for hypothetical symmetric nuclei in the two-dimensional hydrodynamical model were taken arbitrarily to be $1 \%$ for all mass numbers.

The "exact asymptotic values" here refer to the determination of the coefficients in eq. (4) in the limit $A \rightarrow \infty$ for symmetric systems $(N=Z)$ without Coulomb 
TABLE 1

Coefficients of the LDM expansion eq. (4) obtained from fits to theoretical SkM* breathing-mode energies and incompressibilities $K(A)$ (see text for details)

\begin{tabular}{|c|c|c|c|c|c|}
\hline & $\begin{array}{c}K_{\mathrm{V}} \\
(\mathrm{MeV})\end{array}$ & $\begin{array}{c}K_{\mathrm{S}} \\
(\mathrm{MeV})\end{array}$ & $\begin{array}{c}K_{\Sigma} \\
(\mathrm{MeV})\end{array}$ & $\begin{array}{c}K_{\text {curs }} \\
(\mathrm{MeV})\end{array}$ & $x_{\text {rel }}^{2}$ \\
\hline \multicolumn{6}{|l|}{ scaling } \\
\hline exact asymptotic values & 217 & $-209 \pm 2$ & & $-105 \pm 10$ & \\
\hline \multicolumn{6}{|l|}{ 2-parameter fit to $20 \leqslant A \leqslant 200$} \\
\hline$(N=7$, no Coulomb) & $\left.217^{a}\right)$ & -217 & & -57 & 0.006 \\
\hline \multicolumn{6}{|l|}{ 4-parameter fit to eq. (4) } \\
\hline$(90 \leqslant A \leqslant 208)$ with Coulomb & 215 & -209 & -255 & -58 & 0.007 \\
\hline \multicolumn{6}{|l|}{ 2-dim. hydrodyn. } \\
\hline \multicolumn{6}{|l|}{ 3-parameter fit on nuclei } \\
\hline \multicolumn{6}{|l|}{$N=Z$, Coulomb off, with } \\
\hline (i) $40 \leqslant A \leqslant 20000$ & 204 & 0 & & -1236 & 35 \\
\hline (ii) $140 \leqslant A \leqslant 20000$ & 206 & -188 & & -19 & 0.12 \\
\hline (iii) $200 \leqslant A \leqslant 20000$ & 208 & -224 & & 171 & 0.06 \\
\hline (iv) $600 \leqslant A \leqslant 20000$ & 211 & -311 & & 729 & 0.01 \\
\hline \multicolumn{6}{|l|}{ 4-parameter fit via eq. (3) } \\
\hline$(90 \leqslant A \leqslant 208)$ & 212 & -352 & -315 & 649 & 0.17 \\
\hline
\end{tabular}

i) Enforced value.

The 4-parameter fit of the scaling approximation to eq. (4) - with $K_{\text {Coul }}$ fixed - was obtained using 16 spherical nuclei from ${ }^{90} \mathrm{Zr}$ to ${ }^{208} \mathrm{~Pb}$, which include 11 isotopes of $\mathrm{Sn}$ from $A=112$ to $A=132$, two isotopes of $\mathrm{Sm}(A=144,148)$ and ${ }^{\mathrm{I} 40} \mathrm{Ce}$. The 4-parameter fit to the 2-dimensional hydrodynamical model energies was obtained using eq. (3). The 2- and 3-parameter fits were done to theoretical values of $K_{\wedge}$ obtained for nuclei with $N=Z$ omitting the Coulomb interaction (ref. ${ }^{19}$ )).

interaction. The quoted error bars in these coefficients are due to the uncertainty in extracting them from calculations with very large nucleon numbers ( $A$ up to $20000)^{\star}$. For comparison, we also show the results of a two-parameter fit carried out on finite nuclei ( $20 \leqslant A \leqslant 200$ ) with $N=Z$ and the Coulomb force switched off, for both the interactions $\mathrm{SkM}^{*}$ (table 1) and SIII (table 2). By enforcing the value of $K_{\mathrm{V}}$ in these fits, effective values of $K_{\mathrm{S}}$ and $K_{\text {curv }}$ have been obtained. It can be seen that $K_{\mathrm{s}}$ thus obtained is very close to the exact asymptotic one both for $\mathrm{SkM}^{*}$ and SIII, whereas the coefficient $K_{\text {curv }}$ is slightly different from its theoretical value reflecting the absence of higher-order terms and the use of only a limited number of sample nuclei. The results of a four-parameter fit to eq. (4) on $K_{\mathrm{A}}$ obtained via eq. (3) from the scaling energies (shown in fig. 1) of finite and realistic nuclei are also shown for comparison. It can be noticed that the most significant of the coefficients, $K_{\mathrm{V}}$ and $K_{\mathrm{S}}$, are practically identical to the exact asymptotic values as well as to the results of the two-parameter fit. Also the curvature coefficient so obtained compares well with that of a two-parameter fit corresponding to finite nuclei. The asymmetry coefficients obtained in the four-parameter fit are also shown

* See, e.g., ref. ${ }^{23}$ ) for details of determining asymptotic coefficients of a LDM type expansion for the nuclear masses. 
TABLE 2

Coefficients of the LDM expansion eq. (4) obtained from fits to theoretical SIII energies in the scaling approach (as in table 1)

\begin{tabular}{|c|c|c|c|c|c|}
\hline & $\begin{array}{c}K_{V} \\
(\mathrm{MeV})\end{array}$ & $\begin{array}{c}K_{\mathrm{S}} \\
(\mathrm{MeV})\end{array}$ & $\begin{array}{c}K_{2} \\
(\mathrm{MeV})\end{array}$ & $\begin{array}{l}K_{\text {curv }} \\
(\mathrm{MeV})\end{array}$ & $x_{\mathrm{red}}^{2}$ \\
\hline \multicolumn{6}{|l|}{ sculing } \\
\hline $\begin{array}{l}\text { exact asymptotic values } \\
\text { 2-parameter fit to } 20 \leqslant A \leqslant 200\end{array}$ & 356 & $-361 \pm 3$ & & $-116+10$ & \\
\hline $\begin{array}{l}(N=Z, \text { no Coulomb) } \\
\text { 4-parameter fit to eq. (4) }\end{array}$ & $\left.356^{\circ 1}\right)$ & -371 & & -72 & 0.004 \\
\hline$(90 \leqslant A \leqslant 208)$ with Coulomb & 353 & -366 & -379 & -68 & 0.003 \\
\hline
\end{tabular}

i) Enforced value.

in tables 1 and 2 . Scaling thus fulfills the necessary condition for the validity of the LDM expansion (4), and the coefficients $K_{\mathrm{V}}$ and $K_{\mathrm{s}}$ obtained from the fits are seen to be practically equal to the asymptotic ones.

The results of fits of eq. (4) to the hydrodynamical values ${ }^{19}$ ) of $K_{A}$ are shown in table 1 for the $\mathrm{SkM}^{*}$ force. As a consequence of the A-dependent coupling of surface to bulk (parameter $\beta$ ) discussed above, the LDM coefficients are different from those of the scaling case. For values of $A$ below $200, \beta$ depends strongly on

TABL.F. 3

Coefficients of the LDM expansion eq. (4) obtained from fits to the experimental breathing-mode energies from KVI, Groningen ${ }^{12.13 .21}$ ) shown in fig. 1, including ${ }^{27} \mathrm{Mg}$ of ref. ${ }^{25}$ ) The values indicated by ${ }^{31}$ ) are held fixed in the fits. Part (A) shows the fits using the prescription ${ }^{15}$ ) of including the width of strength distribution in the scaling model and (B) shows the fits of the centroid energics in general consideration of eq. (3)

\begin{tabular}{|c|c|c|c|c|c|c|}
\hline & & $\begin{array}{c}K_{Y} \\
(\mathrm{MeV})\end{array}$ & $\begin{array}{c}K_{s} \\
(\mathrm{MeV})\end{array}$ & $\begin{array}{c}K_{2} \\
(\mathrm{MeV})\end{array}$ & $\begin{array}{c}K_{\mathrm{car}} \\
(\mathrm{MeV})\end{array}$ & $x_{n=1}^{2}$ \\
\hline \multicolumn{7}{|c|}{ (A) Including the width of strength distribution } \\
\hline (i) & $\mathrm{Sn}$ and $\mathrm{Sm}$ nuclei & $310 \pm 35$ & $-797 \pm 152$ & $-345 \pm 162$ & $\left.375^{i}\right)$ & 0.07 \\
\hline (ii) & Nuclei of set $(i)+{ }^{20 x} \mathrm{~Pb}$ & $311 \pm 37$ & $-802 \pm 162$ & $-345 \pm 177$ & $375^{\prime \prime}$ & 0.06 \\
\hline (iii) & Nuclei of set $(\mathrm{ii})+{ }^{24} \mathrm{Mg}$ & $301+22$ & $-754 \pm 80$ & $-323 \pm 182$ & $375^{: 13}$ & 0.10 \\
\hline (iv) & Nuclei of set $($ iii $)+{ }^{3 / k} \mathrm{Zr}$ & $300 \pm 23$ & $-751 \pm 83$ & $-316 \pm 192$ & $375^{41}$ & 0.17 \\
\hline$(v)$ & $\begin{array}{l}\text { Constrained fit for nuclei } \\
\text { of set (iv) }\end{array}$ & $217 *)$ & $-177 \pm 85$ & $-206 \pm 173$ & $-621 \pm 285$ & 0.87 \\
\hline \multicolumn{7}{|c|}{ (B) Without the width of strength distribution } \\
\hline (i) & $\mathrm{Sn}$ and $\mathrm{Sm}$ nuclei & $303 \pm 33$ & $-783 \pm 143$ & $-339 \pm 157$ & $375 \%$ & 0.07 \\
\hline (ii) & Nuclei of set $(i)+{ }^{2 n x} \mathrm{~Pb}$ & $306+35$ & $-797 \pm 150$ & $-337 \pm 170$ & $\left.375^{\prime \prime}\right)$ & 0.09 \\
\hline (iii) & Nuclei of set (ii) $+{ }^{24} \mathrm{Mg}$ & $296 \pm 22$ & $-748 \pm 78$ & $-315 \pm 181$ & $375 " 1$ & 0.13 \\
\hline (iv) & Nuclei of set (iii) $+{ }^{4 k} \mathrm{Zr}$ & $295 \pm 22$ & $-748 \pm 82$ & $-310 \pm 185$ & 375 ) & 0.17 \\
\hline
\end{tabular}

a) Entorced value. 
$A$; only for very large nuclei it becomes constant. Therefore, the fits including small nuclei result in high relative $\chi^{2}$ values and quite dramatic variations of the curvature coefficient, reflecting the bad (or even completely missing) convergence of expansion (4). Nevertheless, our fits display a remarkable stability of the volume incompressibility $K_{\mathrm{V}}$, which is our main concern, and which is in satisfactory agreement with the theoretical value. This comes even out of the four-parameter fit to the energies of the two-dimensional hydrodynamical calculations, for which the mass parameters are different from the scaling case and thus eq. (3) a priori does not hold.

\subsection{FITS TO EXPERIMENTAL DATA}

The fits to the experimental data of breathing-mode energies from Groningen ${ }^{12,13,21}$ ) including new precision data ${ }^{13}$ ) on several nuclei are shown in table 3. These fits include data on ${ }^{24} \mathrm{Mg}$ from ref. ${ }^{25}$ ), in which case a strength of about $100 \%$ of the monopole sum rule was found to be exhausted. In the analysis of breathing mode data in ref. ${ }^{13}$ ) the scaling assumption was always used. Thus, the prescription of Treiner et al. ${ }^{15}$ ) of including the width of the strength distribution of the giant monopole resonance, where the centroid energy $E$ in eq. (3) is modified to $E^{\prime}$ by $E^{\prime 2}-E^{2}+3(T / 2.35)^{2}$, was applied. The results of fits using this procedure are shown in table $3(\mathrm{~A})$. Alternatively, we do not, however, want to make the assumption of scaling directly and start with the hypothesis that eq. (3) is valid beyond scaling. The results of such fits are given in table $3(\mathrm{~B})$. The values of parameters thus extracted are only $1-2 \%$ lower than the ones obtained with scaling (table $3(\mathrm{~A})$ ) for a given set of nuclei.

In sets (i) of table $3(\mathrm{~A})$ and (B), we have fitted breathing-mode energies of five $\mathrm{Sn}$ and two $\mathrm{Sm}$ isotopes (fig. 1). The error bars on $K_{\mathrm{V}}$ and $K_{\mathrm{S}}$ are large. Including ${ }^{211} \mathrm{~Pb}$ in the fits, as done in sets (ii), the coefficients remain about the same. Including

${ }^{24} \mathrm{Mg}$ furthermore, as in sets (iii), reduces the error bars in the volume and surface terms considerably. This is due to the increase in the range of the $A^{-1 / 3}$ variation which helps resolve the volume and surface terms more accurately. It is significant that even a light nucleus like ${ }^{24} \mathrm{Mg}$ fits well with the other nuclei. The $K_{\mathrm{V}}$ extracted from the general assumption is $296 \pm 22 \mathrm{MeV}$, which is only marginally different from the conclusion of $300 \pm 23 \mathrm{MeV}$ of the scaling assumption. The surface term is quite large and the asymmetry term shows a good stability at $\sim(-320 \pm 180) \mathrm{MeV}$. The fits also including the new datum ${ }^{12}$ ) for ${ }^{90} \mathrm{Zr}$ are shown in sets (iv). It is gratifying to note that this datum from Groningen fits very well to the trend of earlier data ${ }^{13}$ ) and that the conclusion about $K_{V}$ remains the same, i.e., $\sim 300 \mathrm{MeV}$. In all these fits, the curvature term has been kept fixed at a reasonable value of $375 \mathrm{MeV}$ giving rise to good fits. Varying $K_{\text {curv }}$ between 350 and $400 \mathrm{MeV}$ influences the results for $K_{\mathrm{V}}$ only by about $1 \%$ and $K_{\mathrm{S}}$ by $\sim 4 \%$, whereas $K_{\mathrm{I}}$ remains nearly unaffected. Constraining $K_{\mathrm{V}}$ to $217 \mathrm{MeV}$ in fits (set $(\mathrm{v})$ in table $3(\mathrm{~A})$ ), yielded a small surface and a large curvature term, with a relative $\chi^{2}$ value 5 times larger than the best fit. 
The $\chi^{2}$ value shown in table 3 are relative $\chi^{2}$ values as defined earlier. Reducing the error bars by a factor of 2 in the breathing-mode energies, the corresponding $\chi^{2}$ values go up by a factor of 4 , however, without influencing the values of the coefficients obtained. The factor of 5 difference in relative $\chi^{2}$ between the best fit (free variation) of set (iv) and the constrained fit ( $K_{\mathrm{V}}$ fixed to $217 \mathrm{MeV}$ ) of set (v) can best be illustrated by the quality of the fit to the empirical data as shown in fig. 2 below.

If the LDM expansion (4) is valid and converging sufficiently fast, the reduced quantity $\bar{K}_{\mathrm{A}}$ defined by

$$
\bar{K}_{\mathrm{A}}=K_{\mathrm{A}}-K_{\mathrm{z}}\left(\frac{N-Z}{N+Z}\right)^{2}-K_{\text {coul }} Z^{2} A^{-4 / 3}-K_{\text {curv }} A^{-2 / 3}\left(\approx K_{\mathrm{V}}+K_{\mathrm{S}} A^{-1 / 3}\right),
$$

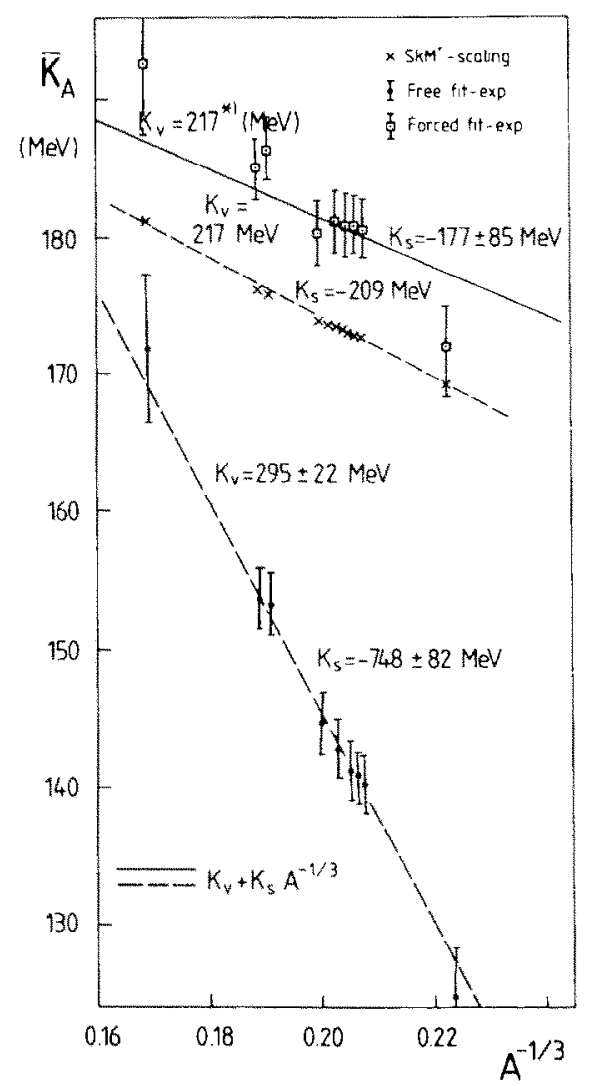

Fig. 2. $\widetilde{K}_{A}$, eq. (9), extracted from the experimental and the theoretical $\$$ SkM data given in fig. 1 with the use of eqs. (3) and (4). The parameters $K_{V}$ and $K_{t}$, indicated at the lines follow from the fit procedure (see also tables 1 and 3 ). The fits to the experimental data include ${ }^{34} \mathrm{Mg}$ from ref. ${ }^{35}$ ), not shown in the figure. The symbols with error bars represent $\bar{K}_{\text {A }}$ extracted from the empirical data by two fits: free fit (* connected by dashed line) and constrained fi (- within $\square)$. The fit with $K_{v}$ constrained to $217 \mathrm{MeV}$ (indicated by $\left.{ }^{*}\right)$ ) is shown by solid line. 
plotted versus $A^{-1 / 3}$, must be close to a straight line intercepting the $\bar{K}_{\mathrm{A}}$ axis at $K_{\mathrm{V}}$ with a slope equal to $K_{\mathrm{S}}$. For $\bar{K}_{\mathrm{A}}$ in eq. (9), the values of $K_{\Sigma}$ and $K_{\text {curv }}$ used were obtained from best fits; for $K_{\text {coul }}$ we used the theoretical value of eq. (5) as described in sect. 3. In fig. 2 the scaling case for $\mathrm{SkM}^{*}$ is given by the dashed line fitting the crosses. It shows how well expansion (4) converges for scaling, in that all the points practically lie on a straight line.

Empirically, the coupling mode between surface and bulk vibrations is known not to be the same for all nuclei. We can, however, check indirectly to what degree this condition is fulfilled by looking at the stability of the output coefficients if the number of input data is varied. We found stability of the coefficients against changes of the number of input data [ the same did not hold ${ }^{22}$ ) if we included older monopole data found in the literature]. The details of the results of fits carried out on several sets of new and old experimental data have been discussed in ref. ${ }^{22}$ ).

Another consistency check arises from the plot of the semi-empirical $\bar{K}_{\mathrm{A}}$ values for the best fit of set (iv) in table 3(B). All data are lying practically on a straight line. Also shown in fig. 2 are the $\bar{K}_{\mathrm{A}}$ points obtained from the experimental data by a fit with an imposed value of $K_{\mathrm{V}}=217 \mathrm{MeV}$ (corresponding to a relative $\chi^{2}=0.87$ of set $(v)$ in table $3(A)$ ). The badness of this fit (shown by the solid line) is reflected also by the fact that the corresponding points do not lie on a straight line. In conclusion, a value of $K_{V} \simeq 217 \mathrm{MeV}$ appears to be ruled out by these data.

\section{Discussion and conclusion}

Since the empirical breathing-mode energies for medium and light nuclei are lower than those resulting from $\mathrm{SkM}^{*}$ calculations (see fig. 1), one would naively expect the empirical value for $K_{\mathrm{V}}$ to be even smaller than the $\mathrm{SkM}{ }^{*}$ value of $217 \mathrm{MeV}$. However, from the different slopes of the lines in fig. 2 the importance of the surface parameter $K_{\mathrm{S}}$ is stressed, which empirically is found to be much larger in magnitude than in the $\mathrm{SkM}^{*}$ case. This also shows that the assumption made in ref. ${ }^{15}$ ), namely that $K_{\mathrm{S}} \simeq-K_{\mathrm{V}}$, and the postulated possibility of a one-parameter fit is not justified in reality. This is apparently a property of Skyrme interactions in the scaling approach as can be seen from tables 1 and 2. If one joins the two data points used by Blaizot ${ }^{11}$ ) (shown by triangles in fig. 1) by a hypothetical line, it would be closer to the data points of $\mathrm{SkM}^{*}$. The $K_{\mathrm{x}}$ derived ${ }^{11}$ ) on the basis of these data is not different from that of $\mathrm{SkM}^{*}$. We emphasize that the conclusion of Blaizot ${ }^{11}$ ) of $K_{x}=210 \pm 30 \mathrm{MeV}$, on the basis of the above two points, was correct. It is, however, no longer compatible with the new experimental situation as depicted by figs. 1 and 2 . The $K_{\wedge}$ values themselves are not much different in theory and in experiment, but disentangling $K_{\mathrm{A}}$ into the volume and surface parts, the importance of $K_{\mathrm{S}}$ in experiment is now revealed.

The inclusion of nuclei smaller than $A \sim 90$ does not give rise to problems in the fits to the scaling energies; there is practically no change in the resulting parameters. 
For the hydrodynamical results ${ }^{19}$ ), however, the fits including $A<90$ result in a $\chi^{2}$ value an order of magnitude larger than for the scaling case; $K_{V}$ does not change much, but $K_{\mathrm{S}}$ changes drastically. On the other hand, if one includes ${ }^{24} \mathrm{Mg}$ in the fit to the cmpirical data (table 3 ), the fit stays as good as without this nuclcus ${ }^{13}$ ). The $\chi^{2}$ value is still much better than for the fit of the hydrodynamical results (case (i) in table 1). We therefore conclude that in reality, the coupling of surface to bulk might be less $A$-dependent than suggested by the theoretical two-dimensional hydrodynamical model we used.

In summary, we have demonstrated the validity of the semi-empirical fitting procedure to determine the first two coefficients of the LDM expansion (4) from a sample of real nuclei. It has been shown that from a fit to a set of new precision data, it becomes possible to disentangle $K_{\Lambda}$ into a volume and a surface part. We found $K_{\mathrm{V}} \sim 300 \pm 25 \mathrm{MeV}$, and a very important surface coefficient $K_{\mathrm{S}} \sim$ $(-750 \pm 80) \mathrm{MeV}$ with relatively small errors (see table 3$). K_{\mathrm{A}}$ as a whole is not much different from the values given in previous theoretical analyses, but $K_{\mathrm{V}}$ and $K_{s}$ are. We point out that there is no Skyrme type force known to us with such a large surface coefficient $K_{\mathrm{S}}$. Correlations in the nuclear surface not describable in the Skyrme/mean-field approximation might be responsible for this large surface term. We further stress that our conclusions rely sensitively on the precision of the recent Groningen data ${ }^{13}$ ) shown in fig. 1 . As shown in the same figure, the hydrodynamical calculations ${ }^{19}$ ) with the Skyrme force SkM* (having $K_{\mathrm{x}}=217 \mathrm{MeV}$ ) reproduce these data within $1 \mathrm{MeV}$ or less. It is the removal of this discrepancy which, in the present phenomenological approach, leads us to the above values of $K_{\mathrm{v}}$ and $K_{\mathrm{s}}$.

There remains the important task to interpret the value of the volume coefficient $K_{\mathrm{V}}$. As discussed in this paper, in both the scaling and the extended hydrodynamical models used in our calculations, $K_{V}$ can be identified with the infinite nuclear-matter incompressibility $K_{x}$. (at zero temperature, and at the saturation density). However, the arguments given in refs. ${ }^{15.16}$ ) leading to $K_{A}^{R}$, eq. (1), show that the volume coefficient of (4) can be different from $K_{x}$. Also, in our fitting procedure using eq. (3) we have explicitly assumed the inertial parameter of the breathing mode to be equal to that of the simple scaling mode, namely $m\left\langle r^{2}\right\rangle$ which could only be proven by a detailed empirical determination of transition densities.

With these reservations in mind, one might tentatively identify the empirical value of $K_{\mathrm{V}}$ with the nuclear-matter incompressibility $K_{\mathrm{x}}$ at saturation. If this conclusion were correct, a new determination of the Skyrme-force parameters would be indicated in order to yield our empirically determined values of both $K_{\mathrm{V}}$ and $K_{\mathrm{S}}$.

With reference to the implications of our result for the equation of state of dense nuclear matter ${ }^{1.2,7}$, we should like to note that an extrapolation of the $K_{x}$ value extracted from breathing-mode analyses to densities two times or more than the saturation value is not possible as long as the functional form of the equation of state is unknown. 
Finally, from general theoretical grounds, the nuclear matter incompressibility must be expected to be energy and frequency dependent, similarly as the effective nucleon mass or the optical potential. (Note that such an energy dependence is not present in any of the scaling, extended hydrodynamical or microscopic RPA calculations with Skyrme forces discussed in this paper). As a consequence, one has to distinguish between a statical and a dynamical incompressibility (see e.g. also ref. $\left.{ }^{26}\right)$ ). Clearly, the dynamical value extracted from giant nuclear monopole vibrations in the energy domain $13-20 \mathrm{MeV}$ need not coincide with the statical one describing ground-statc properties of finite nuclei or nuclear matter.

One of the authors (M.M.S.) thanks Prof. Jorrit de Boer for his hospitality, support and continued encouragement. We are grateful to J.M. Pearson for critical comments and extended discussions on the manuscript. This work was supported by the Alexander von Humboldt Foundation and by BMFT grant No. 06 LM 171.

\section{References}

1) E.A. Baron, J. Cooperstein and S. Kahana, Phys. Rev. Lett. 55 (1985) 126;

J. Cooperstein and E. Baron, preprint BNL-42378; to appear in Supernovae, ed. A. Petschek (Springer, New York, 1989)

2) N.K. Glendenning, Phys. Rev. Lett. 57 (1986) 1120; Phys. Rev. C37 (1988) 2713

3) M. Brack, C. Guet and H.-B. Håkansson, Phys. Reports 123 (1985) 275

4) G. Co' and J. Speth, Phys. Rev. Lett. 57 (1986) 547

5) J.M. Cavedon et al., Phys. Rev. Lett. 58 (1987) 195

6) J. Bartel, G. Wenes, M. Waroquier and J. Ryckebusch, Mod. Phys. Lett. A1 (1987) 509

7) R. Stock, Pliys. Reports 135 (1986) 259;

H. Stöcker and W. Greiner, Phys. Reports 137 (1986) 277

8) J.P. Blaizot, D. Gogny and B. Grammaticos, Nucl. Phys. A265 (1976) 315

9) N. Marty, M. Morlet, A. Willis, V. Comparat and R. Frascaria, Nucl. Phys. A238 (1975) 93

10) O. Bohigas, A.M. Lane and J. Martorell, Phys. Reports 51 (1979) 276

11) J.P. Blaizot, Phys. Reports 64 (1980) 171

12) W.T.A. Borghols, Ph.D. thesis (1988) Univ. Groningen (unpublished);

W.T.A. Borghols, S. Brandenburg, J.H. Meier, J.M. Schippers, M.M. Sharma, A. van der Woude, M.N. Harakeh, A. Lindholm, L. Nilsson, S. Crona, A. Håkansson, L.P. Ekström, N. Olsson and R. De Leo, Nucl. Phys. A504 (1989) 263

13) M.M. Sharma, W.T.A. Borghols, S. Brandenburg, S. Crona, A. van der Woude and M.N. Harakeh, Phys. Rev. C38 (1988) 2562;

M.M. Sharma, in Proc. Int. Winter Meeting on nuclear physics, Bormio (Italy), No. 63 (1988) p. 510, University of Milano

14) V.R. Pandharipande, Phys. Lett. B31 (1970) 635

15) J. Treiner, H. Krivine, O. Bohigas and J. Martorell, Nucl. Phys. A371 (1981) 253

16) B.K. Jennings and A.D. Jackson, Phys. Reports 66 (1980) 141

17) M. Brack and W. Stocker, Nucl. Phys. A388 (1982) 230

18) M. Brack and W. Stocker, Nucl. Phys. A406 (1983) 413

19) P. Gleissl, M. Brack, J. Meyer and P. Quentin, Ann. of Phys., in press

20) R.C. Nayak, J.M. Pearson, M. Farine, P. Gleissl and M. Brack, to be published.

21) S. Brandenburg. W.T.A. Borghols, A.G. Drentje, L.P. Ekström, M.N. Harakeh, A. van der Woude, A. Hăkansson, L. Nilsson, N. Olsson, M. Pignanelli and R. De Leo, Nucl. Phys. A446 (1987) 29 
22) M.M. Sharma, in Proc. Int. Summer School on nuclear astrophysics, La Rabida (Spain), 1988 Nuclear astrophysics, research reports in physics, ed. M. Lozano et al. (Springer, 1989) p. 306

23) W. Stocker, P. Gleissl and M. Brack, Nucl. Phys. A471 (1987) 501

24) Louis Lyons, Statistics for nuclear and particle physics (Cambridge Univ. Press, 1986)

25) II.J. Lu, S. Brandenburg, R. De Leo, M.N. IIarakeh, T.D. Poelhekken and A. van der Woude, Phys. Rev. C33 (1986) 1116

26) W. Stocker, Nucl. Phys. 255 (1975) 121;

1. Speth, private communication (1988) 